\title{
Uncommon Presentation of a Preauricular Sinus
}

\author{
Georgia Alexandra Spyropoulou ${ }^{1,2}$, Alexander Dionyssopoulos ${ }^{2}$, Dimitrios Oikonomou $^{1}$, Guy Sterne ${ }^{1}$ \\ ${ }^{1}$ Department of Plastic and Reconstructive Surgery, City Hospital, Birmingham, UK; ${ }^{2}$ Department of Plastic Surgery, Faculty of \\ Medicine, Aristotle University of Thessaloniki, Thessaloniki, Greece. \\ Email: adionys@auth.gr, gspyropoulou@hotmail.com,droikonomou@yahoo.co.uk, guy.sterne@swbh.nhs.uk
}

Received April 6 ${ }^{\text {th }}, 2012$; revised May $2^{\text {nd }}, 2012$; accepted June $4^{\text {th }}, 2012$

\begin{abstract}
The preauricular sinus is a common finding in the pediatric population. An unusual case of a preauricular sinus that had as only manifestations recurrent postauricular abscesses is presented. A 13 year-old boy presented to the outpatients department with an infected cystic lesion and a hypertrophic scar below the left earlobe. He was operated 7 times in the past by different surgeons; however the postauricular abscess always recurred. Thorough clinical examination revealed a preauricular pit. The combined excision of the preauricular sinus and the remnants of the postauricular cyst resolved the problem and the patient has been asymptomatic ever since during a two years follow-up. Recurrent postauricular infected cysts may, albeit uncommonly, indicate an aberrant preauricular sinus, and the clinician needs to be aware of this possibility.
\end{abstract}

Keywords: Sinus; Preauricular; Cyst; Abcess

\section{Introduction}

Preauricular sinuses were first described by Heusinger in 1864 [1]. They are congenital malformations that result from defective or incomplete fusion of the six auricular hillocks [2]. They are more frequent in Taiwan 1.6\% $2.5 \%$ and some areas of Africa $4 \%-10 \%$ and less frequent in United States $0.1 \%-0.9 \%$ and England 0.9\% [3]. One third of the patients remain asymptomatic and require no treatment [2]. However, once a preauricular sinus becomes infected, its excision is mandatory.

Postauricular abscesses can result from infected epidermoid cysts or rarely type I anomalies of the first branchial cleft [4]. The differential diagnosis includes inflamed adenopathy [5] mastoiditis and subperiosteal abcesses although in the latter two cases auditory and vestibular symptoms usually coexist [4].

We present the case of one patient that had recurrent postauricular abscesses for which he was operated seven times by different surgeons. Finally, thorough clinical examination revealed a preauricular pit. The combined excision of the preauricular sinus and the remnants of the postauricular cyst resolved the problem and the patient has been asymptomatic ever since during our two years follow-up.

\section{Case Report}

A 13 year-old boy presented to the outpatients department with an infected cystic lesion and a hypertrophic scar below the left earlobe (Figure 1).

A review of his medical history revealed recurrent infections of this area approximately every two months since he was 5 years old. The patient was operated seven times in the past for excision of the cystic lesion by two Head and Neck surgeons and two Plastic Surgeons. The biopsies of the initial operations revealed trichilemmal cysts, and the biopsies of the two last operations features in keeping with a hypertrophic scar and ruptured epidermal cyst. In between operations, the patient had recurrent infections and was treated conservatively with antibiotics and in three cases with incision and drainage. He was also referred to the dermatologists for an area $<1 \mathrm{~cm}$ in diameter below the left earlobe with oozing and yellowish crusts that was considered to be seborrheic dermatitis.

Careful clinical examination revealed a preauricular pit slightly anterior to the intertragal notch (Figure 2). A sinogram was requested but failed because the radiologist was unable to cannulate the sinus. A subsequent CT and MRI scan demonstrated no definite extension of the sinus much beneath the subcutaneous tissues, and that a plane existed between the postauricular cyst and the adjacent parotid gland.

During the operation, methylene blue injection (Figure 3 ) into the preauricular sinus rapidly filled the postauricular sinus and confirmed continuity between them. The entire area was carefully dissected under loupe magnification and a wedge of tragal cartilage was excised to reveal a $1 \mathrm{~cm}$ preauricular epithelialised debris 


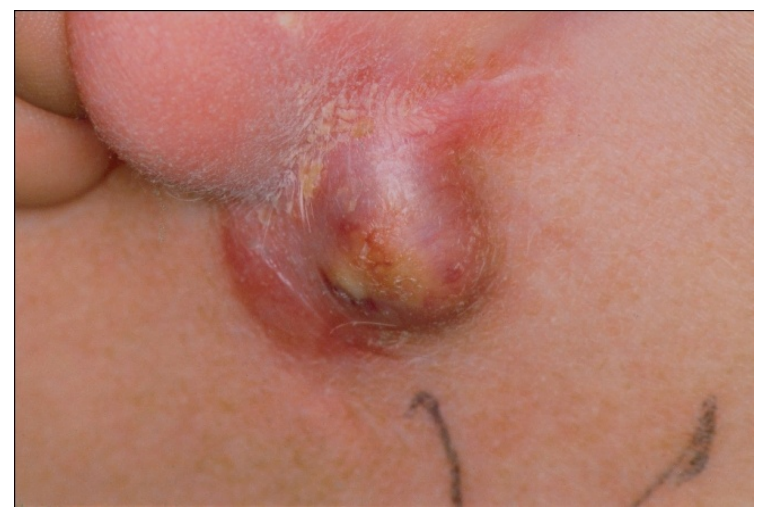

Figure 1. The patient presented to our outpatients department with an infected cystic lesion and a hypertrophic scar below the left earlobe.

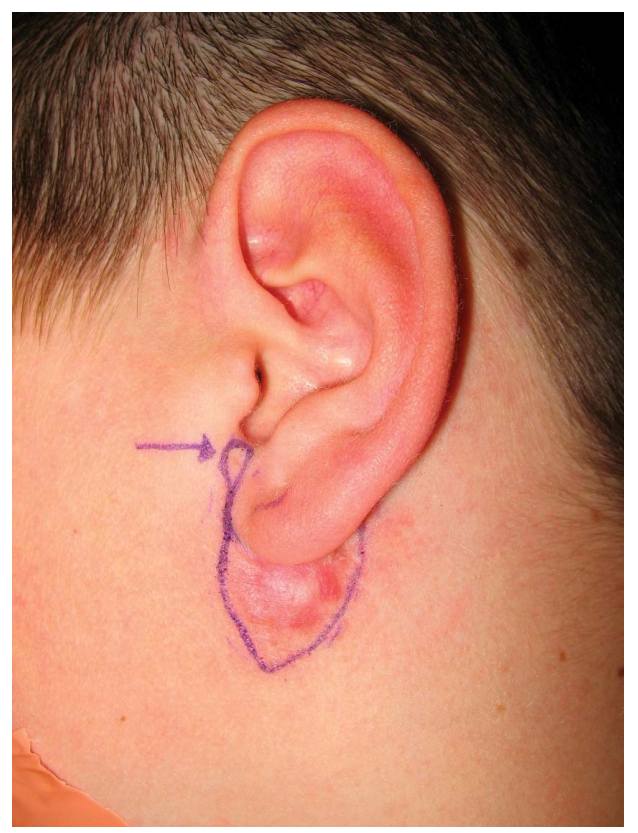

Figure 2. Careful clinical examination revealed a preauricular pit (tip of the black arrow).

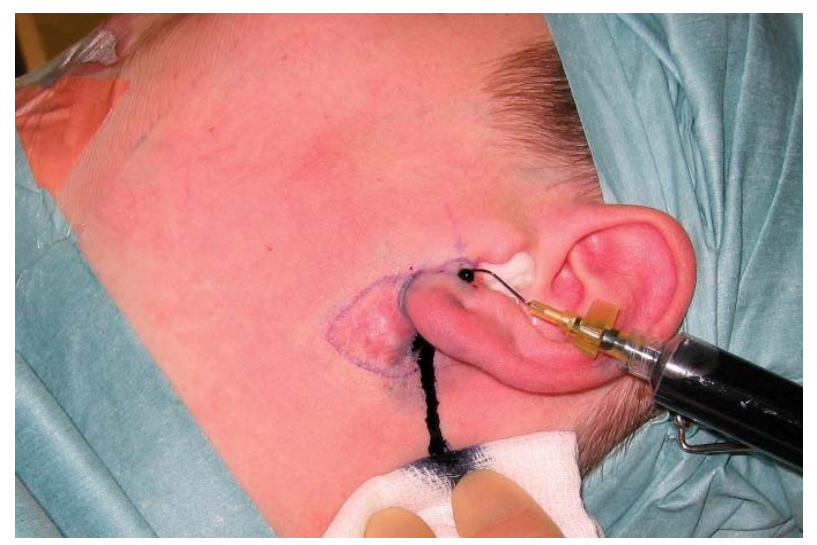

Figure 3. Cannulation of the preauricular sinus and methylene blue injection intraoperatively. Methylene blue pours out from the postauricular sinus. filled sinus cavity. The specimen was excised en block including the wedge of tragal cartilage. The histology report proved excision of inflamed pre- and postauricular sinus with attached keloid scar.

A wedge of tragal cartilage was also excised to ensure removal of the tract's attachments to the perichondrium. The patient has been asymptomatic since the operation and has no nuisances apart from a hypertrophic scar during our two year follow up (Figure 4).

\section{Discussion}

The external ear develops from six mesenchymal prolixferations (hillocks): three from the caudal edge of the $1^{\text {st }}$ branchial arch and another three from the cephalic edge of the $2^{\text {nd }}$ branchial arch. The tragus and the anterior crus of the helix margin develop from the $1^{\text {st }}$ arch, and the rest of the auricle is formed from the $2^{\text {nd }}$ arch [5].

The three predominant theories for the development of preauricular sinuses are: 1) Possible incomplete fusion of the $1^{\text {st }}$ arch hillocks; 2) Isolation of ectodermal folds during auricle formation; and 3) Defective closure of the most dorsal part of the first branchial cleft [6].

Preauricular sinuses and anomalies of the first branchial cleft can have similar presentations. Preauricular sinuses usually present with an opening anterior to the ascending limb of the helix. Occasionally, they occur along the lateral or posterior surface of the helicine crus and the superior posterior margin of the helix, the tragus, or the lobule [7]. First branchial cleft type I anomalies

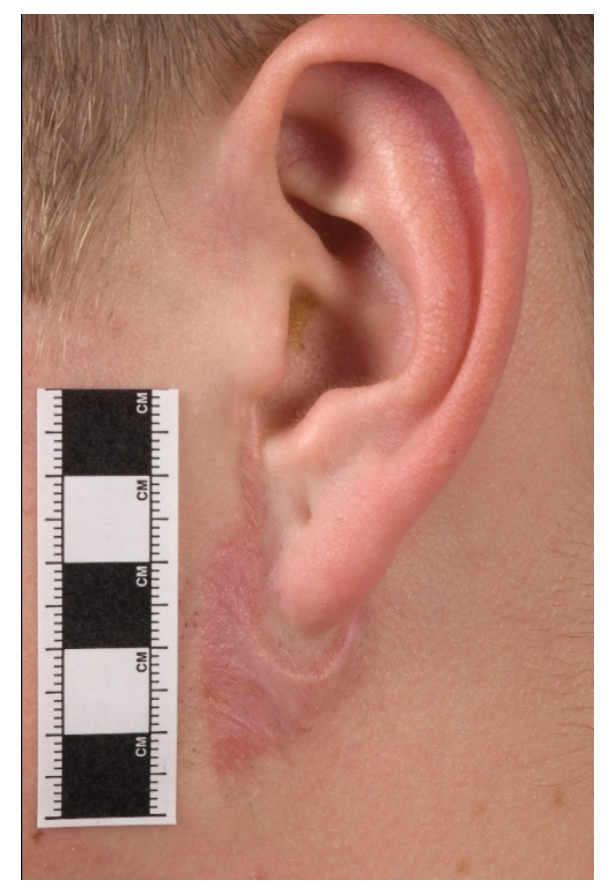

Figure 4. Two years postoperatively, the patient has no nuisances apart from a hypertrophic scar at the incision site. 
can present as a cyst or sinus within the ear canal or postauricular area, whilst an opening in the cheek, below the angle of the mandible, or upper neck above the hyoid bone, suggests a type II anomaly [8]. In the current case, a postauricular cyst was present. However, an opening in the canal was not observed, nor was the course of the tract parallel to the canal. These data suggested that the lesion was a preauricular sinus instead of a branchial cleft type I anomaly [5].

Preauricular sinuses presenting as postauricular inflammation have rarely been reported. Chang and Wu reported three cases presenting as postauricular cysts [4], and Yeo et al. reported 2 cases where the pit was located in the postauricular area in a total of 191 patients [7]. Choi et al. presented a series of 101 patients of which 11 cases with the variant type [5]. They classified the variant types of preauricular sinuses into three categories according to the location of pits: In type 1 the pits were located on the middle area of the crus, in type 2 they were located on the superior area of the crus, and in type 3 on the cymba concha. The present case however, does not fall into any of the aforementioned categories, as the sinus was slightly anterior to the intertragal notch.

If the components of the preauricular sinus are not completely excised recurrence is likely. Therefore, methylene blue injection was used to outline the tracts [2,5] and the operation was performed under loupe magnification [7].

\section{Conclusion}

Recurrent postauricular infected cysts may, albeit uncommonly, indicate an aberrant preauricular sinus, and the clinician needs to be aware of this possibility.

\section{REFERENCES}

[1] H. K. Heusinger, "Hals-Kiemen Finstein von Noch Nicht Beobacheter Form,“ Virchows Archiv, Vol. 29, No. 3-4, 1864, pp. 358-380. doi:10.1007/BF01937182

[2] R. Martin-Granizo, M. C. Pérez-Herrero and A. SánchezCuéllar, "Methylene Blue Staining and Probing for Fistula Resection: Application in a Case of Bilateral Congenital Preauricular Fistulas," International Journal of Oral and Maxillofacial Surgery, Vol. 31, No. 4, 2002, pp. 439-441. doi:10.1054/ijom.2001.0062

[3] I. Aird, "Ear-Pit: Congenital Aural and Preauricular Fistula,” Edinburgh Medical Journal, Vol. 53, No. 9, 1946, pp. 498-507.

[4] P. H. Chang and C. M. Wu, "An Insidious Preauricular Sinus Presenting as an Infected Postauricular Cyst,” International Journal of Clinical Practice, Vol. 59, No. 3, 2005, pp. 370-372. doi:10.1111/j.1742-1241.2005.00437.x

[5] S. J. Choi, et al., "The Variant Type of Preauricular Sinus: Postauricular Sinus,” Laryngoscope, Vol. 117, No. 10, 2007, pp. 1798-1802. doi:10.1097/MLG.0b013e3180caa1ca

[6] P. J. Emery and N. Y. Salama, "Congenital Preauricular Sinus. A Study of 31 Cases Seen over a Ten Year Period," International Journal of Pediatric Otorhinolaryngology, Vol. 3, No. 3, 1981, pp. 205-212. doi:10.1016/0165-5876(81)90004-5

[7] S. W. Yeo, et al., "The Preauricular Sinus: Factors Contributing to Recurrence after Surgery,” American Journal of Otolaryngology-Head and Neck Medicine and Surgery, Vol. 27, No. 6, 2006, pp. 396-400. doi:10.1016/j.amjoto.2006.03.008

[8] Y. C. Nofsinger, et al., "Periauricular Cysts and Sinuses," Laryngoscope, Vol. 107, No. 7, 1997, pp. 883-887. doi:10.1097/00005537-199707000-00009 